\title{
An improved modeling approach for asphaltene deposition in oil wells including particles size distribution
}

\author{
Leila Eyni $^{1}$, Mehdi Assareh ${ }^{2,}$, Shahab Ayatollahi ${ }^{1}$, and Mohammad Ranjbaran ${ }^{1}$ \\ ${ }^{1}$ Sharif Upstream Petroleum Research Institute, Department of Chemical and Petroleum Engineering, Sharif University of \\ Technology, Tehran, Iran \\ ${ }^{2}$ School of Chemical Engineering, Iran University of Science and Technology, Tehran, Iran
}

Received: 21 February 2018 / Accepted: 25 April 2018

\begin{abstract}
There are several approaches to model asphaltene deposition process in the wellbore. There are different assumptions to simplify the problem in the previous investigations for specific conditions, limiting the prediction range of the models. In this work, the effect of precipitated asphaltene particles size is included, to extend the available modeling approaches for deposition profile. To do so, two-dimensional partial differential equations based on asphaltene micro aggregates material balance including asphaltene aggregation, diffusion and deposition are numerically discretized and solved to find asphaltene deposition profile, in radial and vertical directions of vertical oil wells. The modeling results are verified with the results of the well-known $A D E P T$ (asphaltene deposition tool in flow lines) model of Kurup et al. (2011). The size dependent diffusion coefficients of Escobedo \& Mansoori (2010) are used to extend the base model. In addition, the Population Balance Method $(P B M)$ was included to improve the aggregation process description with size distribution of asphaltene particles. Based on the developed model a parametric study is performed to study the effect of asphaltene particles average size, flow rate, wellbore radius and fluid viscosity. The model evaluation shows the importance of asphaltene particle size in the deposition profile. In addition, the evaluation results show that as the average asphaltene particle size increases for a given distribution, the amount of deposition in the wellbore decreases.
\end{abstract}

\section{Introduction}

Asphaltene deposition is an important challenge in the petroleum industry, and proper understanding of its nature and deposition behavior is still under investigation. The general operational definition of asphaltene is according to its solubility in different solvents; Asphaltenes are polar and non-volatile components of the crude oil, which are insoluble in low molecular weight alkanes (e.g. n-heptane). These molecules are slightly soluble in the aromatic solvents (e.g. toluene). Asphaltene deposition in the oil industry processes like production, transportation and processing is one of the important flow assurance problem that can make different challenges. One of the best methods to deal with this problem is the modification of production processes and prevention of the precipitation and deposition by the economical, technical and environmental point of view. In fact, other methods such as chemical and mechanical methods are used when the production process modification is impossible. It should be mentioned that different chemicals at different temperature and pressure

\footnotetext{
* Corresponding author: assarehm@iust.ac.ir
}

conditions have different effects for different kind of crude oils that it makes treatment difficult (Rezakazemi et al., 2017). Therefore, there are many researches about the type of chemicals that are compatible with various crude oils to decrease asphaltene precipitation and then deposition (Hutin et al., 2016; Kashefi et al., 2018).

The fluid flow in porous media mostly occurs in the horizontal directions with a limited change in thermodynamic conditions. In contrast, the petroleum fluid experiences considerable changes in pressure, temperature and composition making challenges to deposition modeling in the vertical wellbores. With upward oil flow in the wellbore, the thermodynamic conditions change leading to the asphaltene separation known as precipitation for which there are many thermodynamic modeling researches in the literature. Deposition is the process of asphaltene aggregates sticking (after precipitation) to the wellbore wall. A review of previous literature reveals that there are only a few qualitative and quantitative research studies on this phenomenon in both laboratory and field scale for the deposition process. The information about the asphaltene deposition position and thickness in the production wells as a function of time are limited. Therefore, model verification is difficult. High-pressure conditions and steep slopes in 
some deviated wells prevent the use of the tools for monitoring of asphaltene deposition. In some cases, the detector itself leads to pressure changes. Therefore, it promotes deposition process due to system instability leading to blockage and sometimes losing the tool.

One of the earliest studies on asphaltene deposition is provided by Ramirez-Jaramillo et al. (2006). The modeling assumptions were in a way that the asphaltene sticks on the walls due to the molecular diffusion in radial direction and the concentration gradient is due to temperature gradient along the wellbore. Based on the results from this model, the deposition thickness cannot exceed $15 \%$ of inner effective diameter of the wellbore. This model fails to match with the recent laboratory data, which shows that, the temperature gradient around the wall does not critically affect the deposition rate. Soltani Soulgani et al. $(2009,2010)$ utilized a simple empirical correlation, and assumed that the deposition rate on the wellbore was controlled by chemical interaction mechanism. Despite the acceptable results obtained from this model, there is no reasonable explanation for the mechanism of the deposition process. The next model was developed by Vargas et al. (2010). This model included some sub-models explaining precipitation, aggregation, deposition and transportation. In fact, according to this model, a deposition simulator was prepared based on a balance equation for turbulent flow associated with thermodynamic precipitation modeling using Perturbed Chain Statistical Associating Fluid Theory $(P C-S A F T)$. Utilizing a simple mathematical model, it predicts the deposition of asphaltene along the flow line in different flow situations (Vargas et al., 2010). The major drawback of this approach was that it assumed a constant rate of precipitation and aggregation along the wellbore. Eskin et al. (2011) developed a model for predicting asphaltene deposition in the wellbore for turbulent flow based on particle mass flux transfer. They studied the deposition problem from fluid mechanic's point of view. In this method, the description of particle transfer into the wall was a function of particle size, density and flow parameters. They used the couette device, consisting of two cylinders, for the verification of their results. The inner cylinder rotates with various speed to apply different flow and particles deposit in the outer immobile wall to model the asphaltene deposition in the wellbore using different particle size distributions with method of particle population balance. This is a strong model from the physical point of view but it has some weaknesses in mathematical simulation. It should be mentioned that in the case of particle size Marques et al. (2008) obtained notable results from laboratory work. In addition, they showed that different sizes of asphaltene aggregates have different aromaticity, aliphatic composition, alkyl chains and metal concentration as well as percent of vanadium to nickel. These results may conclude to the difference in asphaltene deposition rates as well Kurup et al. (2011); Kurup et al. (2012) extended the model proposed by Vargas et al. (2010) through the $1 D$ steady and pseudo steady precipitation simulator $(A D E P T)$. In this model, the precipitation was obtained as a profile for the whole desired length of the well, which is an advantage over Vargas et al. (2010) model, however, aggregation rate was still constant. In addition, they have claimed that the $1 \mathrm{D}$ modeling along the wellbore is reasonable although, the radial diffusion has a noticeable effect.

This work describes a comprehensive asphaltene deposition simulator with macroscopic simulation approach. Despite its simplicity, it includes all processes that, the asphaltene particles may encounter in the wellbore. It considers amount, depth, and profile of asphaltene deposition along the wellbore by taking into account the changes in pressure, temperature and particle size. Like the earlier works of Vargas et al. (2010) and Kurup et al. (2011), three processes of precipitation, aggregation and deposition are included in this model. In addition, the proposed model in this work includes the role of aggregates' size in the asphaltene deposition. For this purpose, the amount of precipitation, asphaltene particles aggregation rate, and asphaltene deposition rate are investigated separately in the wellbore (these parameters are assumed constant in the previous works). Asphaltene precipitation and aggregation are explained by thermodynamics phase behavior and kinetic studies respectively; however, the deposition of asphaltene is related to a series of mechanisms related to surface characteristics, phase behavior, flow regime and rheological properties of the flow. During the precipitation process, a portion of asphaltene particles become unstable which leads to instability and phase separation. Increasing the tendency of particles to aggregate and further aggregation by collisions to each other form the aggregates which is considered by Population Balance Method $(P B M)$, in this study, in addition to the work of Vargas et al. (2010) and Kurup et al. (2011). In this work, using the proposed modeling approach, a sensitivity analysis for different parameters is presented for particles average size, flow rate, viscosity, and well radius. Laboratory data and other required inputs are gathered from Tavakkoli (2013) and the results were compared with results obtained by $A D E P T$.

\section{Methodology}

The focus in this work is to extend a deposition model according to the work of Kurup et al. (2011) including the effective mechanisms in the macroscopic transportation of asphaltene particles through the wellbore (convective flow of micro aggregates, aggregation, diffusion, precipitation and deposition). This model has the ability to predict the probability, location and thickness of asphaltene deposition in the flow lines for different operating conditions. Alongside the upward flow in a wellbore and with respect to the different factors and probabilities, the precipitated particles stick to each other and the aggregates are formed with different sizes. It is also worth noting that the tendency to aggregate between asphaltene masses for different oil samples and with identical precipitant are different. This issue indicates the importance of the oil composition on the precipitation and aggregation rate. Finally, it is likely that these aggregates deposit on the walls of the wellbore due to the contact with the physical surfaces available in the flow lines or previously deposited 


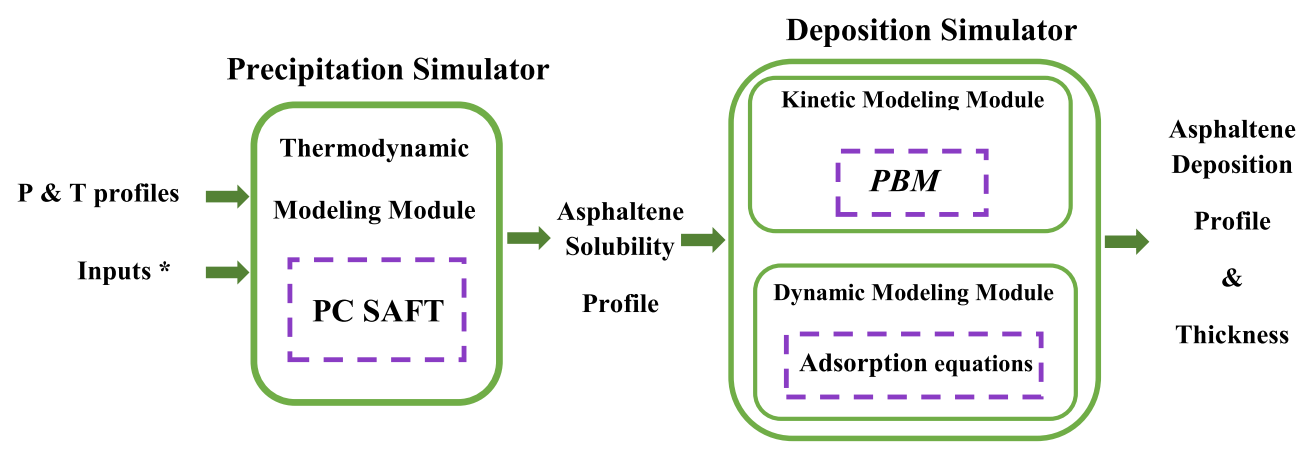

Fig. 1. Schematic diagram of the all steps of developed simulator, Inputs*: Oil \& Gas characterization- Flow rate \& GeometryPrecipitation, Aggregation, and Deposition constant rate- Experimental data

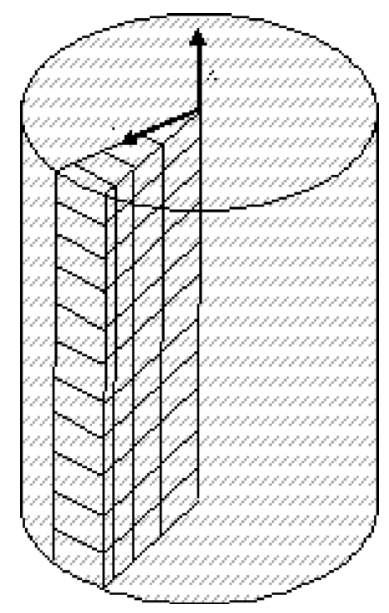

Fig. 2. A schematic of hypothetical discretized well in $2 D$ simulated with finite volume, as in Vargas et al. (2010)

asphaltenes. In other words, without a good understanding of aggregation process, the results for dynamic deposition cannot trusted.

As shown in Figure 1, in this work similar to $A D E P T$ simulator (Kurup et al., 2011), the simulation consists of three modules, thermodynamics, kinetics and dynamics. To obtain amount of asphaltene precipitation along axial length of the wellbore, the pressure and the temperature profiles are obtained through codes for the velocity profile of the two-phase oil and gas flow (velocity calculation for one phase and slippage velocity calculation for the other phase according to flow pattern). The same approach for the calculation of the temperature, pressure and velocity was used in the work of Kurup et al. (2011). Afterwards, for the calculation of soluble asphaltene concentration in equilibrium conditions (i.e., $C_{e q}$ ) in the thermodynamic module, $P C$-SAFT equation is used. It is in the form of dimensionless concentration with respect to the concentration of dissolved asphaltene at inlet conditions. This equation of state has been successfully used by David Ting et al. (2003), Gonzalez et al. (2007), and Vargas et al. (2009) for asphaltene precipitation. This EOS is effective in the study of phase behavior of asphaltene in oil, because of its ability in the modeling of complex molecules like asphaltene in the high pressure and temperature conditions. In $P C$-SAFT equations, characterization of crude oil based on SARA analysis was used to aid in computations reduction. Finally, soluble asphaltene concentration was used as an input for the dynamic module. Before any extension, the approach of Kurup et al. (2011) was developed into $2 D$ using our code. In $2 D$ material balance equation used in this study, different terms were included for precipitation, aggregation, and deposition for each control volume of the wellbore discretized domain (Fig. 2), as in Vargas et al. (2010). Despite its simplicity, this dimensionless equation presents the main processes that asphaltene particles experience:

$$
\begin{aligned}
& \frac{\partial C_{f}}{\partial \xi}+r_{p_{1}}=1, \\
& \underbrace{\frac{\partial C_{i}}{\partial \theta}}_{\text {Accumulation }}=-\frac{60}{49}(\underbrace{1-\eta)^{\frac{1}{7}}}_{\text {Advection }} \frac{\partial C_{i}}{\partial \xi} \\
& +\quad \frac{\partial C_{i}}{\partial \theta} \quad\left(\frac{L}{R}\right) \frac{1}{P e_{i}} \frac{1}{\eta} \frac{\partial}{\partial \eta}\left(\frac{D_{i}}{D_{B i}} \eta \frac{\partial C_{i}}{\partial \eta}\right) \\
& \underbrace{\partial \theta}_{\text {Accumulation }} \underbrace{\partial{ }^{2}}_{\text {Diffusion }} \\
& +r_{A_{i}}+r_{p_{i}} \text {, } \\
& \underbrace{r_{\text {Precipitation }}}_{\text {Aggregation }}
\end{aligned}
$$

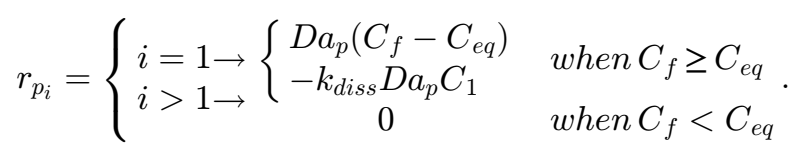

This PDE is subjected to different boundary and initial conditions (Kurup et al., 2011):

$$
B C 1: \frac{\partial C_{i}}{\partial \eta}=0 \text { at } \eta=0 \text { for all } \xi
$$

$$
\begin{gathered}
B C 2: C_{i}=0, C_{f}=1 \text { at } \xi=0 \text { for all } \eta, \\
B C 3: \frac{\partial C_{i}}{\partial \eta}=-D a_{D_{i}} C_{i} \text { at } \eta=1 \text { for all } \xi \\
I C: C_{i}=0 \text { at } \theta=0 \text { for all } \eta, \xi
\end{gathered}
$$


In these relations, $C$ is the dimensionless concentration $\left(c / c_{0}\right)$ of precipitated asphaltene particles. $c_{0}$ is inlet mass concentration of asphaltene solubilized in the oil phase. $i$ is the size of asphaltene particles. $C_{f}$ is dimensionless concentration of asphaltene dissolved in oil phase. $C_{e q}$ is the dimensionless maximum concentration of asphaltene in oil at equilibrium condition. $\theta$ is dimensionless time. $\eta$ is dimensionless radius $(r / R) . r$ is the local radius inside the wellbore and $R$ is the radius of wellbore. $\xi$ is dimensionless length $(z / L) . z$ is local distance alongside the wellbore and $L$ is axial length of the wellbore. $D a_{P}$ is precipitation Damkohler number $\left(D a_{P}\right.$ is calculated without considering size of aggregates), $D a_{D}$ is deposition Damkohler number, and $K_{\text {diss }}$ is kinetic of dissolution factor. The dimensionless parameters are described as:

$$
\begin{gathered}
\mathrm{Pe}=\frac{\text { rate of transport by advection }}{\text { rate of transport by diffusion }}, \\
P e_{i}=\frac{v_{z} R}{D_{B}}
\end{gathered}
$$

$D a_{p}=\frac{\text { rate of production of microaggregate by precipitation }}{\text { rate of transport of microaggregate by advection }}$,

$$
D a_{p}=\frac{k_{P} L}{v_{z}}
$$

$D a_{D}=\frac{\text { rate of consumption of microaggregate by deposition }}{\text { rate of transport of microaggregate by diffusion }}$,

$$
v_{z},
$$

where $P e$ is Peclet number. $\mathrm{v}_{z}$ is average axial velocity of the oil flowing through wellbore, $k_{p}$ is precipitation kinetic constant, $k_{D}$ is deposition kinetic constant, $k_{B}$ is Boltzmann constant, $d_{p}$ is particle diameter, and $v$ is kinematic viscosity.

One of the differences of this study to the work of Kurup et al. (2011) is related to the correlation used for the diffusion term. In the wellbore tubing, there are different flow layers including, turbulent flow core, buffer layer and laminar (wall) sub layer each with different diffusivity coefficients. The asphaltene existing near the tubing wall (i.e., the laminar boundary layer) deposits at a constant rate with the diffusivity coefficient proportional to the distance from the wall. This is another clue for the importance of the $2 D$ modeling. To include it in this research, the correlations presented by Escobedo \& Mansoori (2010) are used:

$$
D_{i}=D_{B}+D_{t} .
$$

In this relation, $D_{B}$ is the Brownian diffusivity and $D_{t}$ is eddy diffusivity. Eddy diffusivity is a function of radial distance, and, is calculated for each of the three main regions in the wellbore, i.e. laminar sub layer, buffer layer, and turbulent core, with different correlations as described in following.

$$
\begin{gathered}
D_{B}=\frac{k_{B} T}{3 \pi \mu d_{p}}, \\
D_{t}=\left\{\begin{array}{l}
v\left(\frac{r^{*}}{11.15}\right)^{3} r^{*} \leq 5 \\
v\left(\left(\frac{r^{*}}{11.4}\right)^{2}-0.1923\right) 5 \leq r^{*} \leq 30 \\
4 v r^{*} 30 \leq r^{*}
\end{array}\right.
\end{gathered}
$$

where:

$$
r^{*}=r\left(\frac{v_{z} \sqrt{f / 2}}{v}\right) .
$$

On the other hand, after applying different diffusivity coefficients for each layer in the wellbore, the velocity in the circular pipe is calculated using turbulent flow distribution function:

$$
v_{z}=v_{z}(r)=\frac{60}{49} v_{z}\left(1-\frac{r}{R}\right)^{\frac{1}{7}} .
$$

In Equation (2), the term on the left-hand side shows changes in the absorbed asphaltene on the wall with time. In this study, it is assumed that all of the absorbed asphaltene will deposit similar to Kurup et al. (2011). The first term on the right-hand side shows the asphaltene particles advection considered only in axial direction, as diffusion is dominant in radial direction. The second term shows asphaltene transport in radial direction under diffusion mechanism. Diffusion in axial direction is not included as the advection is dominant in the axial direction. The third and fourth terms determine the kinetics of aggregation and precipitation, respectively. The deposition process with constant rate is included into the calculations at the third boundary condition. The parameters description in the Equation (2) and the mathematical models used in this work are consistent with the work of Vargas et al. (2010) and Kurup et al. (2011). In the dynamic module, obtaining the particles aggregation kinetic is a prerequisite. However, in this work, this parameter is not constant. Aggregation rate is calculated from $P B M$ for each control volume in the wellbore discretized domain, because, different particle sizes lead to different amounts of absorption. In this work, the geometric PBM form Maqbool (2011) is used. The $r_{A}$ for each class of size is evaluated as following. The mass balance equation was simultaneously solved with equation set describing the size distribution evolution as (Maqbool, 2011): 


$$
\begin{aligned}
r_{A_{i}}= & \frac{K_{i-1, i-1}}{R^{\prime}} C_{i-1}^{2}+C_{i-1} \sum_{j=1}^{i-2} K_{i-1, j} \frac{R^{\prime j-1}}{R^{\prime i-1}-R^{\prime i-2}} C_{j} \\
& -C_{i} \sum_{j=1}^{i-1} K_{i, j} \frac{R^{\prime j-1}}{R^{\prime}-R^{\prime i-1}} C_{j}-C_{i} \sum_{j=i}^{N} K_{i, j} C_{j}
\end{aligned}
$$

where:

$$
\begin{gathered}
K_{i, j}=\frac{k_{i, j} c_{0} L}{v_{z}}, \\
k_{i, j}=\frac{2 R_{g} T}{3 \mu} \frac{\left(d_{i}+d_{j}\right)^{2}}{d_{i} d_{j}} \beta, \\
d_{i}=\left(\frac{R^{\prime i-1}}{\varepsilon}\right) d_{1}, \\
d_{1}=\left(\frac{6 V_{m}}{\pi}\right)^{1 / 3}, \\
V_{m}=\left(\frac{M w_{A} N_{a g g}}{N_{a v} \rho_{o}}\right) .
\end{gathered}
$$

In these relations, $K_{i, i}$ is dimensionless collision kernel between two $i$ th aggregates $\left(K_{i, i}\right.$ is the collision kernel between two $i$ th aggregates). $R^{\prime}$ is geometric scaling factor and is equal to 2.0. $R_{g}$ is universal gas constant and $T$ is absolute temperature. $u$ is viscosity of the medium. $d_{i}$ is the diameter of colliding aggregates $i$ th, $\beta$ is the efficiency of collision, $\varepsilon$ is packing factor for random close packing of spheres, $V m$ is nanoaggregate volume, $M w_{\mathrm{A}}$ is molecular weight of asphaltene, $N_{A}$ is Avogadro constant, $N_{\text {agg }}$ is the number of asphaltene molecules in a nanoaggregate, and $\rho_{0}$ is oil density. In Equation (19), the term in the left-hand side represents the net rate of generation of $i$ th aggregate obtained by the summation of terms for different generation and depletion mechanism. The first and second terms on the right-hand side show the rate of the generation of $i t h$ aggregate, as a result of the reactions of two or more particles with total volume equal to ith aggregate. The third and fourth terms on the right-hand side show the rate of depletion of ith aggregate due to collision with one another or other aggregates. This equation, neglects the breakage and fragmentation terms, otherwise, two adjusting parameters would have been required, making the modeling process more difficult. In addition, the source of precipitation enters the equation continuously and the amount of concentration reduces due to the deposition. The kinetic parameter, $k i, j$ represents the collision kernel between two aggregates with different sizes $\left(\mathrm{m}^{3}\right.$ $\left.\mathrm{kmol}^{-1} \mathrm{~s}^{-1}\right)$. Since it is assumed that nanoaggregates act like primary units for asphaltene aggregations, the Brownian aggregation kernel is used in the calculations. When the pressure changes along the wellbore, the nanoaggregates are destabilized and aggregation produces larger particles. Therefore, the precipitation onset for asphaltene is actually the onset of detection of asphaltene aggregates as they grow to form larger sizes. Besides, with a simplifying assumption, that asphaltene aggregates are spherical and have a packing factor $(\varepsilon)$ which is close to 0.637 for spheres aggregating by accident. Therefore, the only unknown parameter in modeling of $P B M$ is the efficiency of collision $(\beta)$ which depends on the interparticle interactions. The parameter $\beta$, can be estimated from the regression through laboratory data. However, the model is not sensitive to it. Therefore, its value was taken from Maqbool (2011). Finally, after finding $C$, dimensionless concentration of precipitated (phase separated) asphaltene particles, in the wellbore, dimensionless deposition flux, $j_{A}$, is calculated as:

$$
j_{A_{i}}=\left(\frac{\partial C_{i}}{\partial \eta}\right)_{\eta=1}=D a_{D_{i}} C_{i} .
$$

The steady state approach is used to solve the equations. In other words, deposition rate remains constant with the time. Therefore, the pressure and temperature profile and the amount of soluble asphaltene at equilibrium conditions for the entire well length are calculated once. The deposition of asphaltene in the well tubing decreases the available area for the flow and increases the velocity of the flow which is included in the calculation. This leads to pressure drop in tubing, pressure changes and other consequences. However, the mentioned changes can be ignored for more calculation speed without losing the solution accuracy. The wellbore pressure drop, relating to the friction, comprises $10 \%$ of the whole pressure drop along the vertical well. In addition, the asphaltene deposition rate is slow (in the order of $10^{-7}$ ), and therefore it doesn't create an intense change in pressure and temperature. According to Kurup et al. (2012), there is not a significant difference between steady state and pseudo steady state. However, these differences increase with time at the end of forming depositions. Therefore, considering the calculation time, using a steady state method is efficient for the sake of simple and fast modeling. Hence, in this work, it is assumed that the asphaltene deposition on the tubing's wall doesn't change the pressure and temperature profile in the well. It should be noted that, the effect of diameter reduction because of asphaltene deposition is included on reduction of available area for deposition for the next time step, with subtracting asphaltene deposition thickness from the well radius. In this study, after each time step, radius of the wellbore is corrected as:

$$
R\left(t_{n+1}\right)=R\left(t_{n}\right)-\frac{1}{L} \int_{0}^{L} \delta\left(t_{n+1}\right) d z
$$

Where $\delta$ is the height of deposited asphaltene. Its value is obtained as follows:

$$
\rho_{A} \frac{\partial \delta}{\partial t}=\frac{k_{D} R}{2} M w_{A} N_{a g g} \sum_{i=1}^{N} R^{\prime(i-1)} c_{i} .
$$



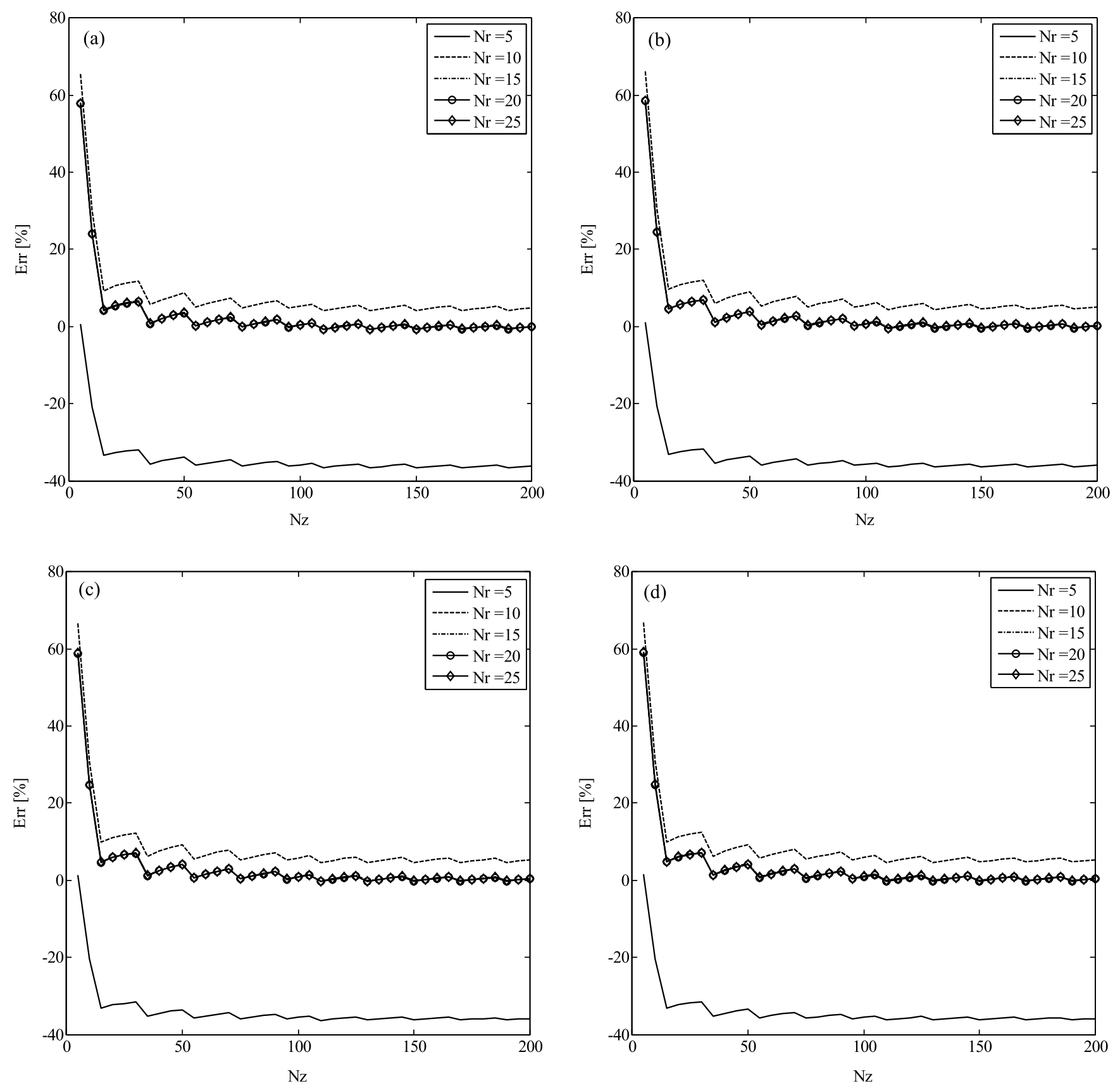

Fig. 3. The relative deviation of average deposition flux for all combinations of $\mathrm{Nz}$ (abscissa) and Nr for different values of $\Delta \mathrm{t}$; (a) $\Delta t=3$ days, (b) $\Delta t=6$ days, (c) $\Delta t=15$ days, and (d) $\Delta t=30$ days.

In addition, this quantity can be obtained from dimensionless parameters according to:

$$
\begin{aligned}
\delta\left(\theta_{n+1}\right)= & \delta\left(\theta_{n}\right) \\
& +\frac{L}{R\left(\theta_{n}\right)} \frac{M w_{A} c_{0}}{\rho_{A}} \frac{\sum_{i=1}^{N} D_{B, i} R^{\prime(i-1)} c_{i}}{v_{z}} \Delta \theta
\end{aligned}
$$

In this work, the well was discretized in radial and axial dimensions and finite volume method was used to solve dimensionless partial differential Equation (Eq. 2). In this model, sensitivity analysis for different parameters were investigated for the particles average size, flow rate, viscosity and well radius.

\section{Results and discussion}

The results description is divided into three main sections. At first, the integrated basic thermodynamic precipitation, and dynamic deposition models are compared with ADEPT results of Kurup et al. (2012) (1D) to evaluate the implementation of the modifications on the data of Tavakkoli (2013). After a verification step, the aggregation 
Table 1. Wellbore fluid composition (Tavakkoli, 2013)

\begin{tabular}{ll}
\hline Component & Reservoir fluid (mol \%) \\
\hline $\mathrm{N}_{2}$ & 0.4 \\
$\mathrm{CO}_{2}$ & 0.92 \\
$\mathrm{C}_{1}$ & 43.4 \\
$\mathrm{C}_{2}$ & 11.02 \\
$\mathrm{C}_{3}$ & 6.55 \\
$\mathrm{iC}_{4}$ & 0.79 \\
$\mathrm{nC}_{4}$ & 3.70 \\
$\mathrm{iC}_{5}$ & 1.28 \\
$\mathrm{nC}_{5}$ & 2.25 \\
$\mathrm{C}_{6}$ & 2.70 \\
$\mathrm{C}_{7+}$ & 26.88 \\
$\mathrm{C}_{7+} \mathrm{Mw}(\mathrm{g} / \mathrm{mol})$ & 228.07 \\
$\mathrm{C}_{7+}$ density $(\mathrm{g} / \mathrm{mol})$ & 0.865 \\
\hline
\end{tabular}

model of $P B M$ is included. Finally, a sensitivity analysis is performed on the asphaltene particles average size, flow rate, viscosity and well radius.

In the stage of model extension in $2 D$ for a single particle size, a mesh independency study was performed and shown in Figure 3. It should be noted to present the sensitivity of the selected mesh to the timestep length, the mesh independency study results are presented for different timesteps. The fine mesh dimensions are $N z=$ $200, N r=50$ and $\Delta t=3$ days. The relative differences of deposited asphaltene of several mesh dimensions form the reference mesh are showed in Figure 3. The mesh with smallest dimensions which does not produce a large deviation was selected for further study. Its dimensions are $N z=40, N r=15 \Delta t=30$ days.

\subsection{Precipitation and deposition modeling verification}

The experimental data of Tavakkoli (2013) were used and the modeling results were compared to his work using ADEPT of Kurup et al. (2012). The wellbore fluid composition is presented in Table 1 . The SARA analysis of the wellbore fluid is in Table 2. The wellbore structure information with all of the used parameters are shown in Table 3. The characterization data for $P C$-SAFT are calculated as shown in Table 4 according to Tavakkoli (2013). The profile of asphaltene content in the equilibrium condition $(\mathrm{Ceq})$ along the wellbore was obtained using vertical flow pattern of the reservoir fluid and compared with the work of Tavakkoli (2013), as shown in Figure 4 (TOP). Density of the reservoir fluid reduces along the tubing with decrease in pressure and temperature. The effect of expansion of the light components of the reservoir fluid is the same as light components injection into the crude oil. Both of them cause asphaltene to precipitate. From precipitation onset, asphaltene starts to precipitate and the precipitation amount increases up to the bubble point. At pressure below the bubble point, light components vaporize gradually out of the oil. This event causes the solubility of asphaltene in the crude oil to increase until
Table 2. SARA analysis for wellbore fluid (Tavakkoli, 2013)

\begin{tabular}{ll}
\hline & SARA analysis of STO (wt \%) \\
\hline Saturate & 68.3 \\
Aromatic & 11.6 \\
Resin & 18.8 \\
Asphaltene & 1.3 \\
\hline
\end{tabular}
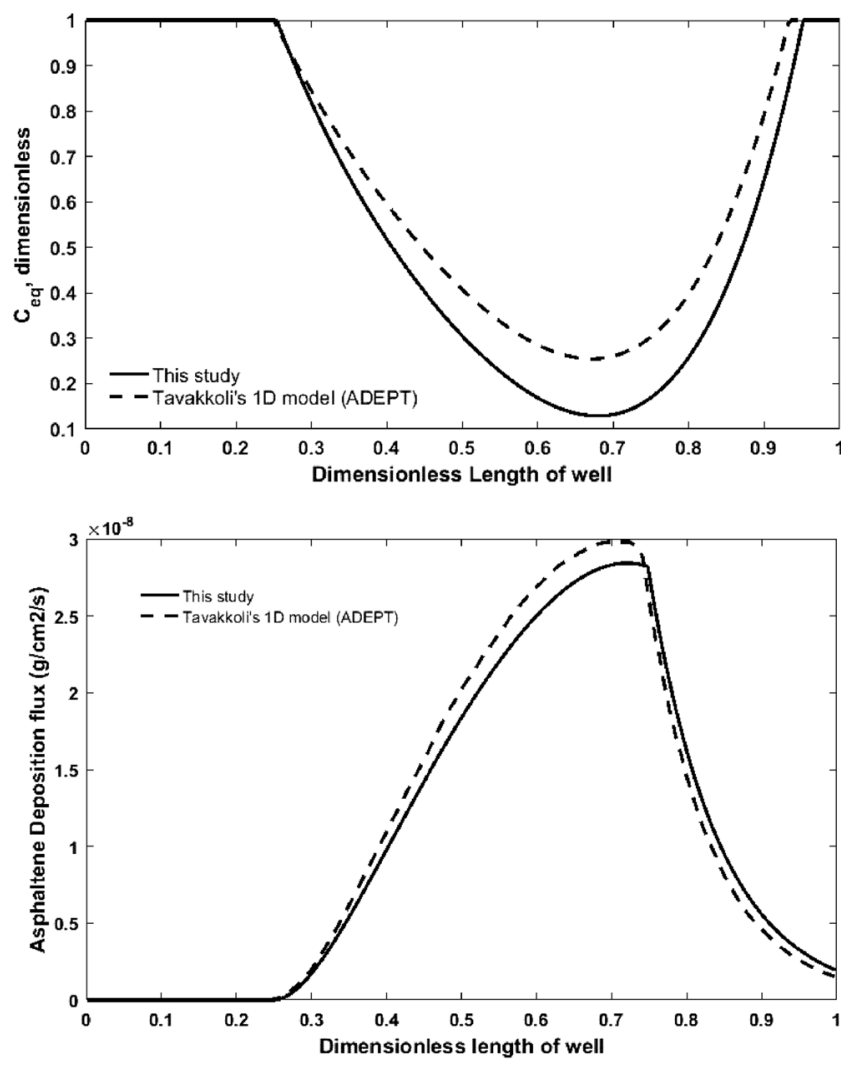

Fig. 4. (TOP) Profile of asphaltene content in equilibrium condition (comparison to (Tavakkoli, 2013)) along the wellbore, (BOTTOM) Asphaltene deposition results of $2 D$ model with constant diffusion coefficient and precipitation model parameters in this work and $1 D$ ADEPT in Tavakkoli (2013) profile along the wellbore

the lower onset point in which asphaltene dissolves again completely. The calculated solubility parameter of the oil supports this speculation (Bahrami et al., 2015). In fact, for each reservoir fluid, there is a minimum solubility point for asphaltene at fluid bubble point as mentioned by Tavakkoli (2013). It should be noted that, according to the figure, the difference(which is not considerable) in the equilibrium concentration between this work and the work of Tavakkoli (2013) is due to the fact that both works made separate regression, of $P V T$ data. In fact, for $P V T$ data in this work, we made the regression process using our code. As the regression is a multivariable optimization, it does not produce unique answer. 
Table 3. Required modeling properties and parameters (Tavakkoli, 2013)

\begin{tabular}{llll}
\hline Reservoir temperature $\left({ }^{\circ} \mathrm{F}\right)$ & 240 & Reservoir pressure $(\mathrm{MPa})$ & 62.05 \\
${ }^{\circ}$ API & 39 & GOR $\left(\mathrm{m}^{3} / \mathrm{m}^{3}\right)$ & 195.90 \\
Length $(\mathrm{ft})$ & 52389 & Diameter $(\mathrm{in})$ & 4.881 \\
Fluid density $\left(\mathrm{g} / \mathrm{cm}^{3}\right)$ & 0.6370 & Deposit density $\left(\mathrm{g} / \mathrm{cm}^{3}\right)$ & 0.9292 \\
Flow rate $(\mathrm{bbl} /$ day $)$ & 13482 & Fluid viscosity $(\mathrm{p})$ & 0.0134 \\
Kinetic constant of Precipitation, $k_{P}\left(\mathrm{~s}^{-1}\right)$ & $1.32 \times 10^{-5}$ & Kinetic constant of Deposition, $k_{D}\left(\mathrm{~s}^{-1}\right)$ & $2.471 \times 10^{-6}$ \\
Inlet concentration $\left(\mathrm{g} / \mathrm{cm}^{3}\right)$ & $6.2 \times 10^{-3}$ & Deposition time $($ day $)$ & 30 \\
Friction factor & 0.018 & Dissolution factor, $k_{\text {diss }}$ & 1 \\
& & & \\
Packing factor & 0.636 & Molecular weight of asphaltene, $M w_{A}(\mathrm{~g} / \mathrm{mol})$ & 1700 \\
\hline
\end{tabular}

Table 4. Characterized Crude Oil (Tavakkoli, 2013)

\begin{tabular}{llllll}
\hline Component & $\mathrm{M}_{\mathrm{w}}(\mathrm{g} / \mathrm{mol})$ & Composition $(\mathrm{wt} /)$ & $\sigma$ & $\mathrm{M}$ & $\varepsilon / \mathrm{k}$ \\
\hline $\mathrm{N}_{2}$ & 28.01 & 0.1899 & 3.3130 & 1.21 & 90.96 \\
$\mathrm{CO}_{2}$ & 44.01 & 0.5374 & 2.7852 & 2.073 & 169.21 \\
$\mathrm{C}_{1}$ & 16.04 & 9.8257 & 3.7039 & 1 & 150.03 \\
Heavy gas & 43.13 & 14.0297 & 3.6042 & 2.0183 & 203.9262 \\
Saturate & 176.43 & 51.5100 & 3.9062 & 5.3959 & 250.3649 \\
Aromatic + Resin $(\gamma=0.029)$ & 277.7 & 22.9268 & 4.0087 & 6.8732 & 290.2926 \\
Asphaltene $(\gamma=0.404)$ & 1700 & 0.9804 & 4.2959 & 30.6616 & 377.6932 \\
\hline
\end{tabular}

The next step, is the deposition calculation. Since all of the deposition model improvements, in this work, are based on the work of Kurup et al. (2011), the correct implementation of the method is verified at first using the data of Tavakkoli (2013). The deposition flux profiles are plotted in Figure 4 (BOTTOM) in comparison to Tavakkoli (2013). The first figure is from $1 D$ (ADEPT simulator) of Kurup et al. (2011) in Tavakkoli (2013), the second is prepared with this work, $2 D$ approach (constant diffusion coefficient) and $C_{e q}$ from thermodynamic precipitation approach of this work. Two figures are similar. This means, asphaltene deposition profile of $2 D$ becomes very close to $1 D$ (ADEPT simulator) of Kurup et al. (2011) in Tavakkoli (2013). The sensitivity analysis shows that the constant diffusion coefficient is so large that, it doesn't show the radial distribution effects. It is one of the reasons of the similarity between $2 D$ and $1 D$ (ADEPT simulator) of Kurup et al. (2011) in Tavakkoli (2013)

In the next step, diffusion coefficients of Escobedo \& Mansoori (2010) were utilized (Eq. 14). The size effect results in different penetration rates for each size and each layer of the wellbore were calculated in $2 D$ model. Therefore, the particles absorb with different rates compared to those come from the center to the layer near the wellbore. According to Figure 5, the deposition profiles along the wellbore are obtained by choosing different sizes for asphaltene particles, which results in different deposition flux. The deposition flux decreases for larger size

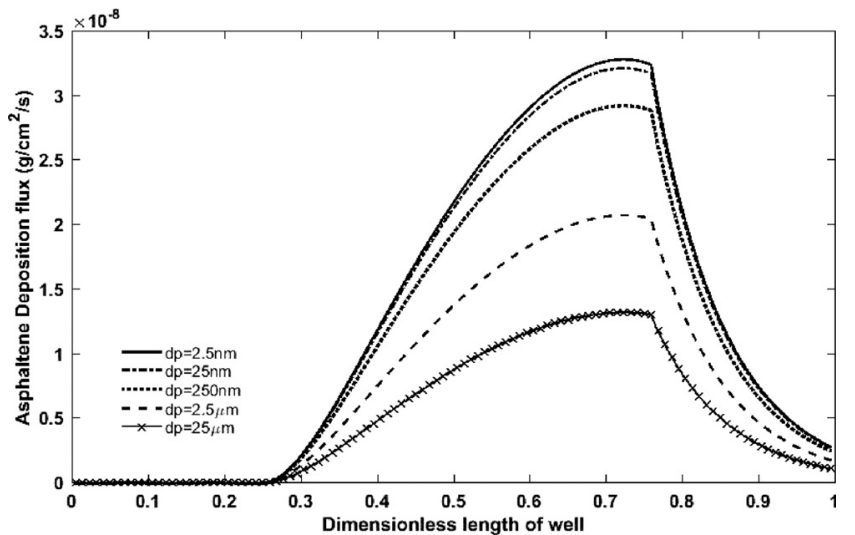

Fig. 5. Asphaltene deposition profile (with the diffusion coefficients of Escobedo \& Mansoori (2010)) along the wellbore for different particle sizes.

distribution of asphaltene particles, which shows the importance of applying the size effect into the absorption equation. Nanometer is the order of magnitude in which asphaltene begins to precipitate (Eskin et al. (2011) showed that this size could be between $1.6-5 \mathrm{~nm}$ ). In addition, Eskin et al. (2011) declared that in simulating asphaltene deposition inside Couette device, particles larger than 10 micrometers do not settle down. This is the reason for choosing this range for the current study. 


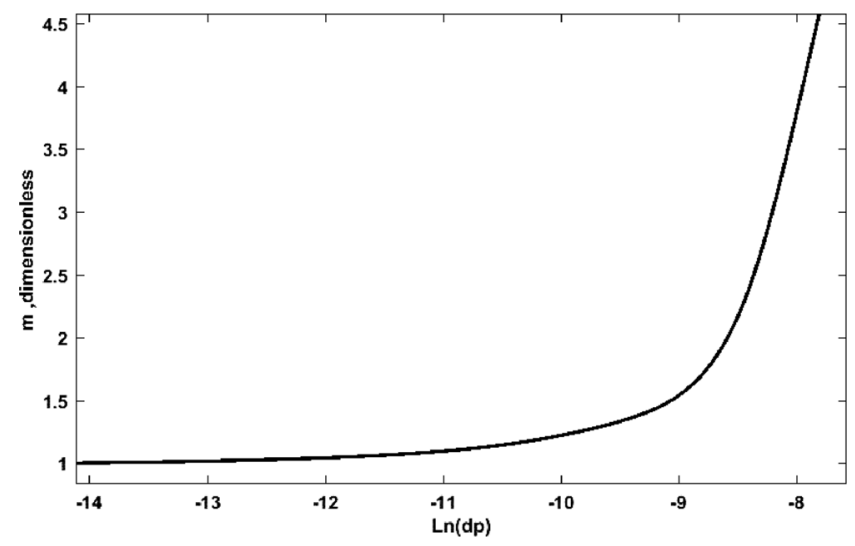

Fig. 6. Asphaltene concentration in the layer near to the wellbore proportional to the average concentration along the radius versus to size of particles. $m$ versus $\mathrm{d}, m=$ Asphaltene concentration in the layer near the wellbore proportional to the average concentration along the radius, $d_{\mathrm{p}}=$ size of particles. Their values were averaged over the whole length of the wellbore.

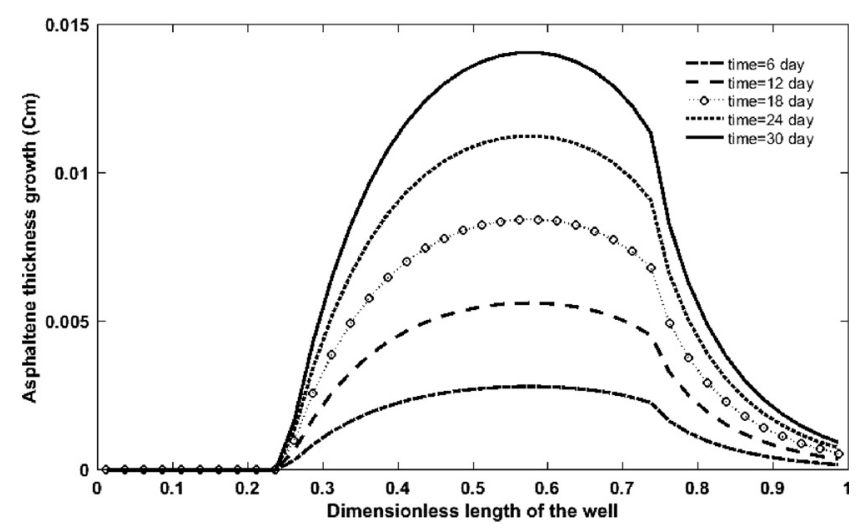

Fig. 7. Asphaltene deposition profile (proposed 2D modeling approach with Escobedo \& Mansoori (2010) size dependent diffusion coefficients and $P B M$ ) along the wellbore for different periods of deposition.

The differences between $2 D$ and $1 D$ simulation, for each particle size, are provided in Figure 6. This figure shows the asphaltene concentration in the layer close to the wellbore (it was obtained when we discretized the well) proportional to the average concentration along the radius versus particle size. Their values were averaged over the whole length of the wellbore. The asphaltene concentration in the layer close to the wellbore and the average concentration along the radius are equivalent to $2 D$ and $1 D$ models, respectively. In fact, the differences between $2 D$ and $1 D$ simulations become larger for the bigger sizes. When the size of the particles is small, the deposition flux on the wall is equal to the average volume flux in that depth of well, and $1 D$ simulation is similar to $2 D$. The deviation becomes greater with size. For the aforementioned reasons, there is no doubt that particle size has critical effect on the diffusion coefficient as well as deposition flux.

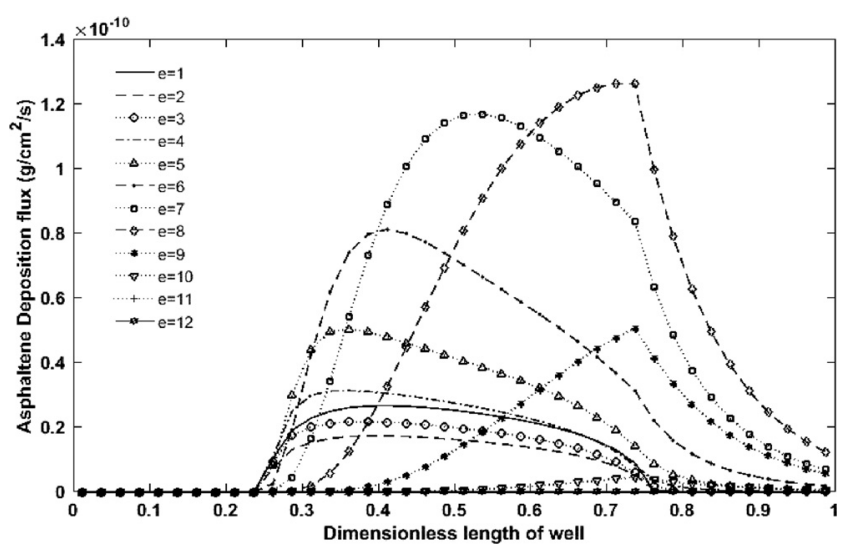

Fig. 8. Asphaltene deposition profile (proposed 2D modeling approach with Escobedo \& Mansoori (2010) size dependent diffusion coefficients and $P B M$ ) along the wellbore for each considered particle sizes; contribution of particles with different size in the deposition flux.

\subsection{Aggregation modeling using $P B M$}

To include different particle sizes effect, the asphaltene particles were classified into 12 classes according to size. Including, the particle sizes bigger than the last class in our classification didn't have any effect on the deposition of asphaltene. Afterwards, the absorption equation (Eq. 2) and population balance (Eq. 19) were simultaneously solved for each size in all of the blocks. As the deposition rate was considered steady and time independent, a number of proper time intervals were selected to reach steady state conditions. In Figure 7, the thickness of deposited asphaltene along the wellbore versus time is shown. As it indicates after one month, the radius of the wellbore reduces about $0.015 \mathrm{~cm}$, which is lower than the model with constant diffusion coefficient and no population balance. Figure 8 shows the contribution of particles with different sizes in the deposition flux. The shape of deposition flux for each size is almost like the general trend. It is shown that, initially, the deposition rate is increased with size and after a specific point, it is inversed. Besides, the results show that the asphaltene particle of class eight has the highest deposition rate. In fact, it is concluded that for this case, size distribution effect is well associated with the effect of size abundance and they cannot be separated. Moreover, the distribution of bigger sizes in the wellbore is higher in this stage of deposition; therefore, increasing in deposition flux is to some extent due to the abundance of those sizes. In Figure 9 , the results of the $2 D$ simulation with population balance are compared with the results of Tavakkoli (2013) ( $1 D$ with constant diffusion coefficient) for 30 days. In this figure, deposition rate and deposition trend in the models are compared. The results clearly indicate that the deposition rate in comparison with $A D E P T$ in Tavakkoli (2013) is smaller. This difference in deposition rate is due to applying the kinetic of aggregation effect (i.e. size distribution) in the simulation. In ADEPT (Kurup et al., 


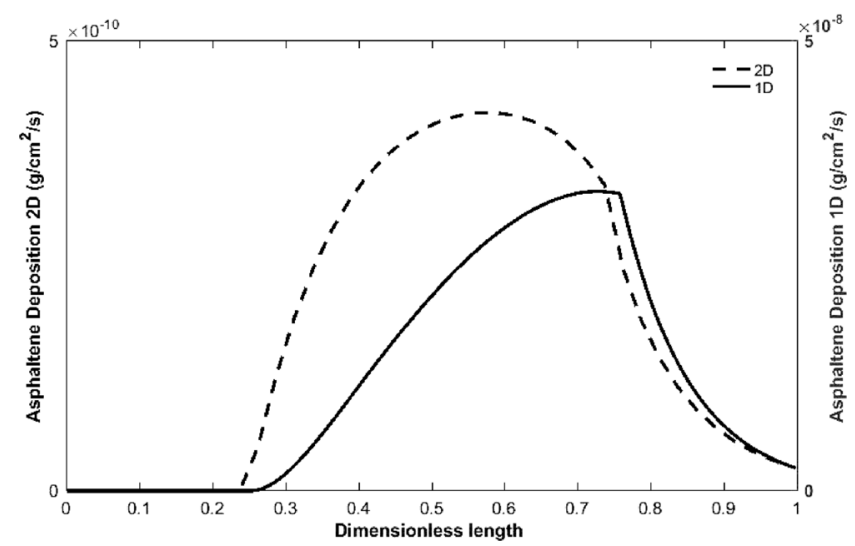

Fig. 9. Comparison of deposition trend of $1 D$ ADEPT Kurup et al. (2011) in Tavakkoli (2013) and this work (proposed 2D approach coupled with $P B M$ ) for 30 days.

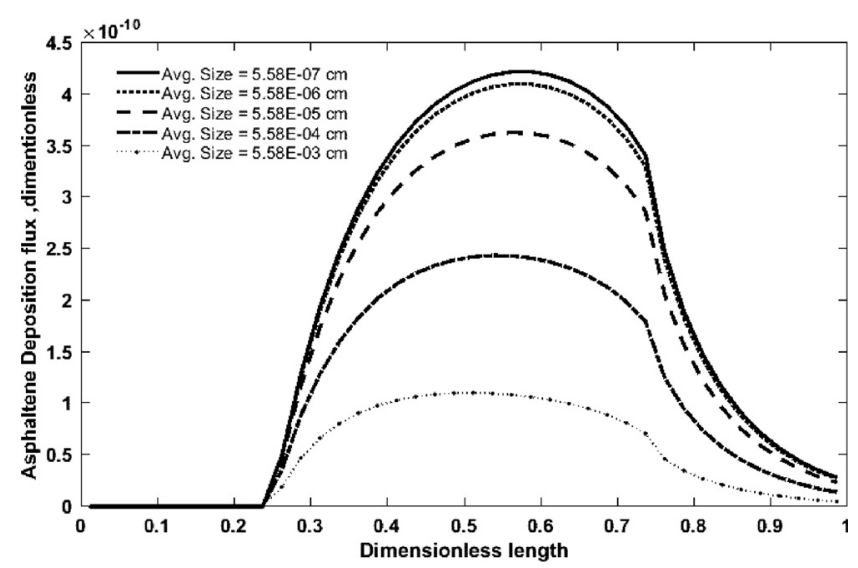

Fig. 10. Asphaltene deposition profile (proposed model) along the wellbore for different average sizes for 30 days.

2011), the amount of the kinetic aggregation constant was considered so small that the probability of sticking the particles to one another was almost zero. As the ADEPT simulation considers the single particle size, the selection of such kinetic aggregation constant is reasonable. Nevertheless, by applying size distribution and assigning the probability of particle sticking, the larger aggregates are formed. As it is mentioned before, the diffusion rate for the particles with larger size is smaller; therefore, it has a lower deposition rate. Thus, the size distribution, with larger particle sizes, leads to a lower deposition rate. The trends of diagrams are similar with the minor difference because of kinetic of aggregation and size distribution effect. In fact, as it was shown, each class of considered size distributions has its own trend and the overall effects results in a distinct trend from $A D E P T$. To show the size effect separately in Figure 10, the deposition flux is plotted for different average sizes for 30 days. The deposition flux decreases with increasing in average size of asphaltene aggregates. It should be mentioned that some of inhibitors, which are used for preventing the deposition of asphaltene, break the bigger



Fig. 11. Average deposition flux versus average amount of different size distributions.

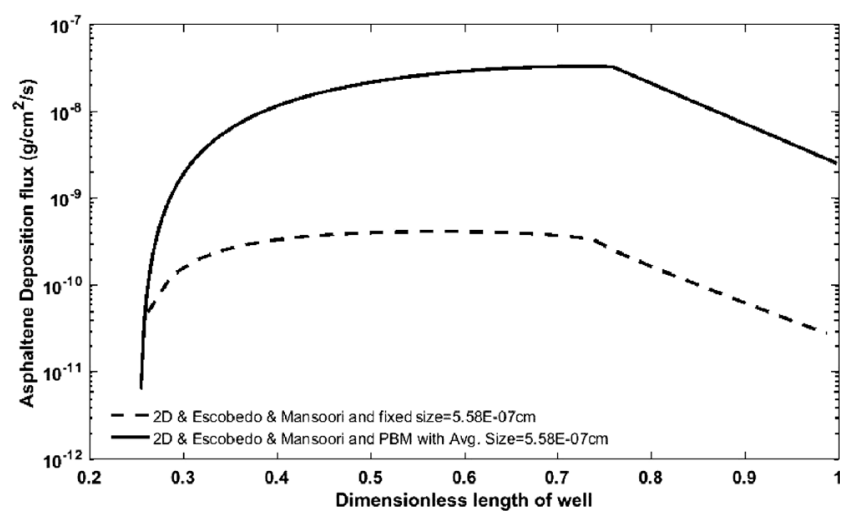

Fig. 12. Comparison of $2 D$ modeling using with fixed particle size (size effect is included on the diffusion coefficient) and $2 D$ modeling using variable particle size $(P B M)$ for 30 days.

asphaltene aggregations and in fact, unlike their injection goals, they lead to asphaltene deposition worsening Vargas et al. (2010). To demonstrate this effect, for average amount of different size distributions, average deposition flux was obtained and plotted in Figure 11. It means that the deposition flux starts from a maximum amount and reduces until it reaches its maximum change at a point and then the slop of change reduces so that, finally, it reaches the minimum amount and after that, there is no change in deposition flux.

The importance of $2 D$ modeling with $P B M$ (this work), in which a range of particle sizes is included, over the $2 D$ modeling without $P B M$, in which a fixed size equal to average particle size, is demonstrated for 30 days in Figure 12.

\subsection{Sensitivity analysis}

Using the extended deposition model, the effect of different parameters on the deposition can be analyzed. In this work, a sensitivity analysis of three different flow rates was 


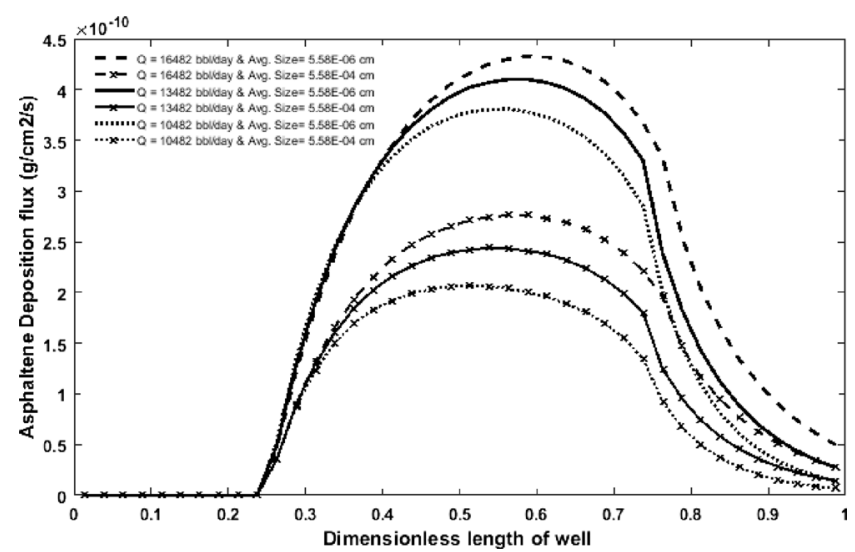

Fig. 13. Sensitivity analysis to the wellbore flow rates for different particle size distributions after 30 days.



Fig. 14. Sensitivity analysis to the viscosity for different particle size distributions after 30 days.

conducted using two different particle size distributions as showed in Figure 13. According to this figure, with increasing the flow rate within the same time (30 days), two effects on deposition flux can be seen; the first one (nonaligned effect) is convection, in which, as it increases, it carries more particle and prevents deposition. Therefore, the amount of deposition increases with increasing the flow rate. This effect is in part clear at the ascending part of the diagram. In second mechanism (aligned effect), velocity gradient in the radial direction increases with flow rate and it leads to increase the concentration gradient in the radial direction (At the beginning, asphaltene particles are generated by precipitation. This is modeled as a volume source. Therefore, all the points in radial direction have the same chance. Suppose asphaltene is precipitated in the wellbore when the onset point is reached. Because of velocity gradient, particles in the middle of the wellbore flow faster than the particles near the wall. Therefore, concentration distribution is stretched. This results in sharp gradients perpendicular to the flow, which diffusion can then iron out. The use of radial gradient in velocity distribution as well as radial diffusion coefficient in the first equation manages this effect.). Then this gradient leads the

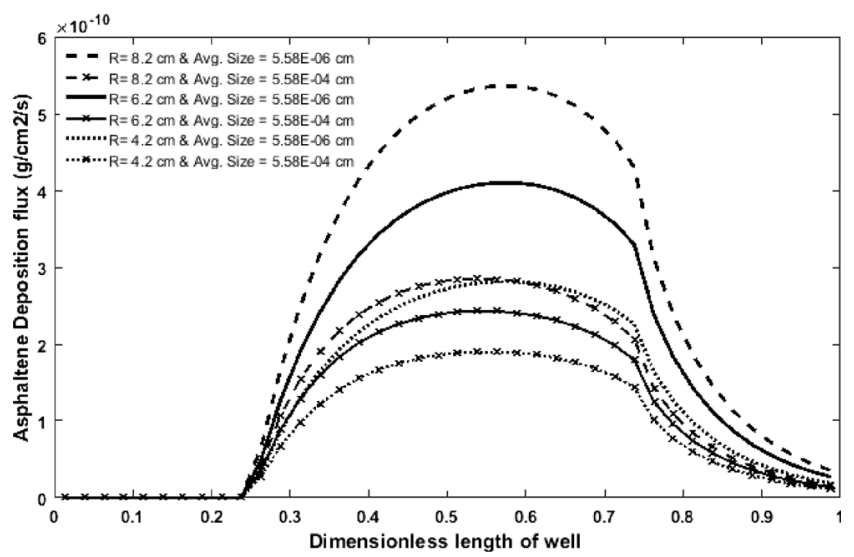

Fig. 15. Sensitivity analysis for the effect wellbore radius for different particle size distributions, deposition profile after 30 days.

particle to move to the wall of wellbore. In fact, the penetration of particles increases toward the wall. The second mechanism prevails the first one in a part of the path. The reason of this dominance is the increase in suspended asphaltene concentration along the well. Therefore, the usage of chokes with smaller opening diameter can help decreasing the deposition. It is worth mentioning that in $1 D$ model of Kurup et al. (2011), only, the effect of first mechanism is investigated. Therefore, it is also another reason for the superiority of the $2 D$ model. The first mechanism in ascending part of figure for the bigger average size of asphaltene aggregates is lower than smaller average size of asphaltene aggregates, which shows that the investigation on the effect of parameters without considering average size of asphaltene aggregates may not be completely correct. Therefore, proposed modeling can enhance our understanding of size effect on deposition. After that, a similar sensitivity analysis was conducted for two different particle size distributions, for three different viscosities, as showed in Figure 14. The viscosity influences two parameters, diffusion and kernel, in which, the former and the latter have favorable and adverse effects on deposition promotion, respectively. As it is mentioned before, diffusion does not show the radial distribution effects. In fact, it reduces the mass transfer resistance in the direction of the well radius. Hence, the amount of deposition increases with increasing diffusion. Besides, the aggregation increases with the kernel. Therefore, the amount of deposition increases with decreasing the kernel. Due to the aforementioned reasons, with increasing the viscosity within the same time (30 days), the deposition flux increases in the smaller average size of asphaltene aggregates. However, with increasing the average size of asphaltene aggregates, the mutual effects of the viscosity and average size of asphaltene aggregates on deposition can be seen. Once again, it shows that the investigation on the effect of parameters without considering average size of asphaltene aggregates may not be completely correct. In the next step, another sensitivity analysis was conducted for three different well radiuses at two different particle size distributions, as showed in Figure 15. The flow rate 
increases with the radius in a constant velocity. In addition, as we showed before, the amount of deposition increases with increasing the flow rate. Therefore, the amount of deposition increases with decreasing the well radius. Finally, in all sensitivity analyses, the deposition flux decreases with increasing in average size of asphaltene aggregates for each constant flow rate, viscosity, well radius and temperature, which confirms the result of Figure 10.

\section{Conclusion}

In this work, the dynamic deposition of asphaltene in the oil wells including the precipitation, aggregation and deposition of asphaltene with changes in the temperature, pressure, and size along the axial length of the wellbore was simulated and analyzed through an improved modeling approach. The proposed modeling approach extends the available models to $2 D$ numerical simulation including different particle size effects through $P B M$. These three parts were integrated in a simple and comprehensive $2 D$ simulator. The model was verified with a common asphaltene deposition tool to evaluate its basic modeling capabilities to obtain the asphaltene deposition profile along tubing. The analysis showed that larger aggregate sizes, makes significant reduction of the diffusion rate and consequently lower deposition rate. Moreover, the relative importance of the aggregation and deposition phenomena were analyzed through the proposed model including size distribution effect. This result revealed the impracticality or even adverse effect of some asphaltene precipitation inhibitors. Besides, the critical size effect of asphaltene aggregate sizes and mutual effects of flowrate on the deposition process were included in the parametric study. Effects of flowrate were related to the effect of convection and velocity gradient in the radial direction, which the former and the latter have favorable and adverse effects on deposition promotion, respectively. Moreover, effects of the critical size were related to the effect of diffusion and collision kernel, which as we explained in results and discussion section the former and the latter have favorable and adverse effects on deposition promotion, respectively. At the end, a sensitivity analysis of viscosity and radius were conducted using two different particle size distributions. In fact, mutual effects of viscosity and average size of asphaltene aggregates make a different result for various particle size distributions in sensitivity analysis. Therefore, to predict the asphaltene deposition accurately in production wells with pressure and temperature depletion, it is recommended to consider the asphaltene size distribution.

\section{Nomenclature}

C dimensionless concentration of precipitated asphaltene particles

$C_{e q}$ dimensionless concentration of asphaltene in the oil at equilibrium

$C_{f}$ dimensionless concentration of asphaltene solubilized in the oil phase $c_{0} \quad$ inlet concentration of asphaltene solubilized in the oil phase, $\mathrm{mol} / \mathrm{cm}^{3}$

$d_{i} \quad$ the diameter of colliding aggregates $i$ th

$d p \quad$ the particle diameter

$D \quad$ diffusion, $\mathrm{cm}^{2} / \mathrm{s}$

$D_{B} \quad$ Brownian diffusion, $\mathrm{cm}^{2} / \mathrm{s}$

$D_{t} \quad$ turbulence diffusion, $\mathrm{cm}^{2} / \mathrm{s}$

$D_{a p} \quad$ precipitation Damkohler number

$D a_{A}$ aggregation Damkohler number

$D a_{D} \quad$ deposition Damkohler number

$f \quad$ friction factor

$j_{A} \quad$ dimensionless deposition flux

$J_{A} \quad$ deposition flux, $\mathrm{g} / \mathrm{cm}^{2} / \mathrm{s}$

$K_{\text {diss }}$ Kinetic of dissolution factor

$K_{p} \quad$ precipitation kinetic constant, $1 / \mathrm{s}$

$K_{D} \quad$ deposition kinetic constant, $1 / \mathrm{s}$

$K_{B} \quad$ Boltzmann constant, $1.38066 \times 10^{23} \mathrm{~J} / \mathrm{K}$

$K_{i, j}$ dimensionless collision kernel between $i$ th size aggregate and $j$ th size aggregate

$R \quad$ radius of the wellbore, $\mathrm{cm}$

$r \quad$ the local radius inside the wellbore, $\mathrm{cm}$

$R \quad$ geometric scaling factor

$R_{q} \quad$ the universal gas constant, $8.314 \mathrm{~J} / \mathrm{mol} / \mathrm{K}$

$T$ absolute temperature, $\mathrm{K}$

$v_{z} \quad$ average axial velocity, $\mathrm{cm} / \mathrm{s}$

$V \quad$ nanoaggregate volume $\mathrm{cm}^{3}$

$z \quad$ local distance alongside the wellbore, $\mathrm{cm}$

$v \quad$ kinematic viscosity, $\mathrm{cm}^{2} / \mathrm{s}$

$\rho_{0} \quad$ oil density, $\mathrm{g} / \mathrm{cm}^{3}$

$\rho_{A} \quad$ asphaltene density, $\mathrm{g} / \mathrm{cm}^{3}$

$\mu \quad$ viscosity of the medium, $\mathrm{g} / \mathrm{cm} / \mathrm{s}$

$\beta \quad$ the efficiency of collision

$\varepsilon \quad$ packing factor for random close packing of spheres

$\theta \quad$ dimensionless time

$\eta \quad$ dimensionless radius

$\xi \quad$ dimensionless length

$\delta \quad$ the height of deposited asphaltene, $\mathrm{cm}$

\section{References}

Bahrami P., Kharrat R., Mahdavi S., Firoozinia H. (2015). Prediction of the gas injection effect on the asphaltene phase envelope. Oil Gas Sci. Technol. - Rev. IFP Energies nouvelles, 70, 6, 1075-1086.

David Ting P., Hirasaki G.J., Chapman W.G. (2003). Modeling of asphaltene phase behavior with the SAFT equation of state. Pet. Sci. Technol., 21, 3-4, 647-661.

Escobedo J., Mansoori G.A. (2010). Heavy-organic particle deposition from petroleum fluid flow in oil wells and pipelines. Pet. Sci., 7, 4, 502-508.

Eskin D., Ratulowski J., Akbarzadeh K., Pan S. (2011). Modelling asphaltene deposition in turbulent pipeline flows. Can. J. Chem. Eng., 89, 3, 421-441.

Gonzalez D.L., Hirasaki G.J., Creek J., Chapman W.G. (2007). Modeling of asphaltene precipitation due to changes in composition using the perturbed chain statistical associating fluid theory equation of state. Energy Fuels, 21, 3, 1231-1242.

Hutin A., Argillier J.-F., Langevin D. (2016) Influence of pH on oil-water interfacial tension and mass transfer for asphaltenes model oils. Comparison with crude oil behavior. Oil Gas Sci. Technol. - Rev. IFP Energies nouvelles, 71, 58. 
Kashefi S., Lotfollahi M.N., Shahrabadi A. (2018). Investigation of asphaltene adsorption onto zeolite beta nanoparticles to reduce asphaltene deposition in a silica sand pack. Oil Gas Sci. Technol. - Rev. IFP Energies nouvelles, 73, 2.

Kurup A.S., Vargas F.M., Wang J., Buckley J., Creek J.L., Subramani H, Chapman W.G. (2011). Development and application of an asphaltene deposition tool (ADEPT) for well bores. Energy Fuels 25, 10, 4506-4516.

Kurup A.S., Wang J., Subramani H.J., Buckley J., Creek J.L., Chapman W.G. (2012). Revisiting asphaltene deposition tool (ADEPT): field application. Energy Fuels, 26, 9, 5702-5710.

Maqbool T. (2011). Understanding the kinetics of asphaltene Precipitation from Crude oils. The University of Michigan.

Marques J., Merdrignac I., Baudot A., Barré L., Guillaume D., Espinat D., Brunet S. (2008). Asphaltenes size polydispersity reduction by nano- and ultrafiltration separation methods comparison with the flocculation method. Oil Gas Sci. Technol. - Rev. IFP Energies nouvelles, 63, 1, 139-149.

Ramirez-Jaramillo E., Lira-Galeana C., Manero O. (2006). Modeling asphaltene deposition in production pipelines. Energy Fuels 20, 3, 1184-1196.
Rezakazemi M., Mirzaei S., Asghari, M., Ivakpour J. (2017). Aluminum oxide nanoparticles for highly efficient asphaltene separation from crude oil using ceramic membrane technology. Oil Gas Sci. Technol. - Rev. IFP Energies nouvelles 72, 34.

Soltani Soulgani B., Rashtchian D., Tohidi B., Jamialahmadi M. (2009). Integrated modelling methods for asphaltene deposition in wellstring. J. Jpn. Petrol. Inst., 52, 6, 322-331.

Soltani Soulgani B., Rashtchian D., Tohidi B., Jamialahmadi M. (2010). A novel method for mitigation of asphaltene deposition in the wellstring. Iran. J. Chem. Chem. Eng., 29, 2, 131-142.

Tavakkoli M. (2013). Simulation of asphaltene slurry for oil dominant system in vertical wells. (PhD), Sharif University of Technology, Tehran, Iran.

Vargas F.M., Creek, J.L., Chapman, W.G. (2010). On the Development of an Asphaltene Deposition Simulator $\dagger$. Energy Fuels, 4, 24, 2294-2299.

Vargas F.M., Gonzalez D.L., Hirasaki, G.J., Chapman, W.G. (2009). Modeling asphaltene phase behavior in crude oil systems using the perturbed chain form of the statistical associating fluid theory (PC-SAFT) equation of state $\dagger$. Energy Fuels, 23, 3, 1140-1146. 